Citation: Sönmez, D., Yeşilot, G., "On Weakly Prime Fuzzy Ideals of Commutative Rings". Journal of Engineering Technology and Applied Sciences 4 (1) 2019 : 19-25.

\title{
ON WEAKLY PRIME FUZZY IDEALS OF COMMUTATIVE RINGS
}

\author{
Deniz Sönmez* (D), Gürsel Yeşilot \\ $a^{*}$ Department of Mathematics, Faculty of Arts and Sciences, Yildiz Technical University, Turkey \\ dnzguel@hotmail.com (*corresponding author),gyesilot@yildiz.edu.tr
}

\begin{abstract}
In this paper, we present a new notion of fuzzy ideals: called weakly prime fuzzy ideal. Let $R$ be a commutative ring with non-zero identity. A nonconstant fuzzy ideal $\mu$ of $R$ is called weakly prime fuzzy ideal if $0_{t} \neq x_{r} y_{s} \in \mu$ implies $x_{r} \in \mu$ or $y_{s} \in \mu$ for all $t \in(0, \mu(0)]$. We investigate some properties of this notion. Morever, it is established relations between weakly prime ideals and weakly prime fuzzy ideals of commutative rings.
\end{abstract}

Keywords: Weakly prime fuzzy ideals, prime fuzzy ideals

\section{Introduction}

The concept of weakly prime ideals of a commutative ring was introduced and studied by D.D. Anderson and E. Smith in [1]. Subsequently, S. E. Atani and F. Farzalipour introduced the notion of weakly primary ideals [2]. These studies brought a new concept to the literature of ring theory. Here we aim to define the concept of weakly prime fuzzy ideals in fuzzy ring theory.

We assume throughout this paper that all rings are commutative with non-zero identity. Unless stated otherwise $L=[0,1]$ will be a complete lattice. $L I(R)$ denotes the set of fuzzy ideals of R. $\lambda_{I}$ denotes the characteristic function of $I$.

Recall from [1] that a proper ideal $I$ of $R$ is called a weakly prime ideal if whenever $0 \neq a b \in I$ , then either $a \in I$ or $b \in I$. Note that every prime ideal is a weakly prime ideal. However, the converse is not true. Recall from [7] that a fuzzy ideal $\mu$ of $R$ is called fuzzy prime ideal if for any two fuzzy points $x_{r}, y_{s}$ of $R, x_{r} y_{s} \in \mu$ implies either $x_{r} \in \mu$ or $y_{s} \in \mu$. From [7] we know 
that a nonconstant fuzzy ideal $\mu$ is said to be weakly completely prime fuzzy ideal if and only if for any $x, y \in R, \mu(x y)=\max \{\mu(x), \mu(y)\}$.

Motivated from this concept, in Section 2, we define weakly fuzzy ideals and we investigate many properties of weakly prime fuzzy ideals. A nonconstant fuzzy ideal $\mu$ of $R$ is called weakly prime fuzzy ideal if $0_{t} \neq x_{r} y_{s} \in \mu$ implies $x_{r} \in \mu$ or $y_{s} \in \mu$ for all $t \in(0, \mu(0)]$. Among many results in this study, it is proved (Example 2.3) that every prime fuzzy ideal is a weakly prime fuzzy ideal but converse is not true. It is shown that how to construct a weakly prime fuzzy ideal by a weakly prime ideal (Theorem 2.6). Also, it is shown (in Theorem 2.7) if $\mu(0)=1$ then $\mu$ is a weakly prime fuzzy ideal if and only if $\mu_{*}$ is a weakly prime ideal and $|\operatorname{Im} \mu|=2$. In theorem 2.9, we show that a nonconstant fuzzy ideal $\mu$ is a weakly prime fuzzy ideal if and only if $0_{t} \neq A B \subseteq \mu$ implies $A \subseteq \mu$ or $B \subseteq \mu$ for any $A, B$ fuzzy ideals and for all $t \in(0, \mu(0)]$. Morever, we investigate to properties of cartesian product of weakly prime fuzzy ideals. Finally, it is defined partial weakly prime fuzzy ideal and associated with partial weakly prime fuzzy ideals and weakly prime ideals.

\section{Weakly prime fuzzy ideals}

Definition 2.1 A nonconstant fuzzy ideal $\mu$ of $R$ is said to be weakly prime if $0_{t} \neq x_{r} y_{s} \in \mu$ implies $x_{r} \in \mu$ or $y_{s} \in \mu$ for any fuzzy points $x_{r}, y_{s}$ of $R$ and for all $t \in(0, \mu(0)]$.

Note that $0_{t}$ is defined by

where $x \in R$ and $t \in(0, \mu(0)]$.

$$
0_{t}(x)=\left\{\begin{array}{lc}
t, & x=0 \\
0, & \text { otherwise }
\end{array}\right.
$$

Also we can state the definition as $\mu$ is a weakly prime fuzzy ideal if $x_{r} y_{s} \in \mu$ such that $x_{r} \notin \mu$ and $y_{s} \notin \mu$ implies that $0_{t}=x_{r} y_{s}$ for any $t \in(0, \mu(0)]$.

Theorem 2.2 Every prime fuzzy ideal is a weakly prime fuzzy ideal.

Proof. The result follows by the definition of prime fuzzy ideal.

The following example shows a weakly prime fuzzy ideal needs not be prime fuzzy ideal.

Example 2.3 Let $R=Z_{6}$. Then the fuzzy ideal $0_{1}$ is a weakly prime fuzzy ideal that not prime fuzzy ideal. Assume that $x_{r} y_{s} \in \overline{0}_{1}$ such that $x_{r} \notin \overline{0}_{1}$ and $y_{s} \notin \overline{0}_{1}$. Then $r \wedge s \leq \overline{0}_{1}(x y)$ and $r>\overline{0}_{1}(x), s>\overline{0}_{1}(y)$ so $\overline{0}_{1}(x) \wedge \overline{0}_{1}(y)<r \wedge s \leq \overline{0}_{1}(x y)$. Thus we conclude that $0<r \wedge s$, $x, y \neq 0$, and $x y=0$. Thus $x_{r} y_{s}=x y_{r \wedge s}=0_{t}$ for $r \wedge s=t \in(0,1]$. Hence $0_{1}$ is a weakly prime fuzzy ideal. But since $\overline{2}_{1} \overline{3}_{1} \in \overline{0}_{1}$ and neither $\overline{2}_{1} \in \overline{0}_{1}$ nor $\overline{3}_{1} \in \overline{0}_{1}$ then $0_{1}$ is not prime fuzzy ideal.

Theorem 2.4 If $\mu$ is a weakly prime fuzzy ideal then $\mu_{k}$ is a weakly prime ideal of $R$ for all $k \in(0, \mu(0)]$. 
Proof. Assume that $0 \neq x y \in \mu_{k}$ for all $k \in(0, \mu(0)]$ and $x, y \in R$. Since $k \neq 0$ and $0 \neq x y$ then $0_{k}(0)=k \neq 0=(x y)_{k}(0)$ so $0_{k} \neq(x y)_{k}$. If $x y \in \mu_{k}$ then $\mu(x y) \geq k$ and $(x y)_{k}=x_{k} y_{k} \in \mu$. Since $\mu$ is a weakly prime fuzzy ideal and $0_{k} \neq(x y)_{k}=x_{k} y_{k} \in \mu$ then $x_{k} \in \mu$ or $y_{k} \in \mu$. So we get that $x \in \mu_{k}$ or $y \in \mu_{k}$ hence $\mu_{k}$ is a weakly prime ideal.

Note that the converse of the theorem is not true.

Example 2.5 Let $R=Z_{4}$ and $\mu$ be defined by

$$
\mu(x)=\left\{\begin{array}{ll}
\frac{1}{2}, & x \in\{\overline{0}, \overline{2}\} \\
0, & \text { otherwise }
\end{array} .\right.
$$

For all $k \in(0, \mu(0)], \mu_{k}=\{\overline{0}, \overline{2}\}$. Since $\{\overline{0}, \overline{2}\}$ is a prime ideal of $R$ then $\mu_{k}=\{\overline{0}, \overline{2}\}$ is a weakly prime ideal. Now we show $\mu$ is not a weakly prime fuzzy ideal. For fuzzy points $\overline{2}_{\frac{3}{4}}, \overline{3}_{\frac{1}{2}}$ of $R$, $\overline{2}_{\frac{3}{4}} \cdot \overline{3}_{\frac{1}{2}} \neq 0$ for all $t \in(0, \mu(0)]$. Also $0_{t} \neq \overline{2}_{\frac{3}{4}} \cdot \overline{3}_{\frac{1}{2}}=\overline{2}_{\frac{1}{2}} \in \mu$ because $\mu(\overline{2}) \geq \frac{1}{2}$. But $\overline{2}_{\frac{3}{4}} \notin \mu$ and $\overline{3}_{\frac{1}{2}} \notin \mu$ since $\frac{3}{4}=\overline{2}_{\frac{3}{4}}(\overline{2})>\mu(\overline{2})=\frac{1}{2}$ and $\frac{1}{2}=\overline{3}_{\frac{1}{2}}(\overline{3})>\mu(\overline{3})=0$. Hence $\mu$ is not weakly prime fuzzy ideal.

Theorem 2.6 Let $I$ be a weakly prime ideal of $R$ and $1 \neq \alpha \in L$. If $\mu$ is the fuzzy ideal defined by

$$
\mu(x)=\left\{\begin{array}{ll}
1 & x \in I \\
\alpha & x \notin I
\end{array} \text { for all } x \in R\right.
$$

then $\mu$ is a weakly prime fuzzy ideal of $R$.

Proof. Assume that $0_{t} \neq x_{r} y_{s} \in \mu$ such that $x_{r} \notin \mu$ and $y_{s} \notin \mu$. Then $x_{r}(x)=r>\mu(x)$ and $y_{s}(y)=s>\mu(y)$. Then we get that $\mu(x)=\mu(y)=\alpha$ and $x, y \notin I$. Since $0_{t} \neq x_{r} y_{s}$ then there exists an $a \in R$ such that $0_{t}(a) \neq x_{r} y_{s}(a)$.

Case 1 If $a=0$, then $0_{t}(a)=t \neq x_{r} y_{s}(a)$. For all $t \in(0, \mu(0)]$, it must be $x_{r} y_{s}(a)=0$ so $a=0 \neq x y$.

Case 2 If $a \neq 0$, then $0_{t}(a)=0 \neq x_{r} y_{s}(a)$ so $x y=a \neq 0$.

We conclude in all cases that $x y \neq 0$. Since $\mu(x)=\alpha<r$ and $\mu(y)=\alpha<s$ then $\alpha<r \wedge s \leq \mu(x y)$. Thus $\mu(x y)=1$ and $x y \in I$. But this contradicts that $0 \neq x y$ and $x, y \notin I$ because $I$ is a weakly prime ideal. Hence $\mu$ is a weakly prime fuzzy ideal.

Theorem 2.7 Let $\mu$ be a nonconstant fuzzy ideal of $R$ and $\mu(0)=1$. Then $\mu$ is a weakly prime fuzzy ideal of $R$ if and only if $\mu_{*}$ is a weakly prime ideal of $R$ and $|\operatorname{Im} \mu|=2$. 
Proof. Let $\mu_{*}$ be a weakly prime ideal and $\operatorname{Im} \mu=\{\alpha, 1\}$. We show that $\mu$ is a weakly prime fuzzy ideal. By the previous theorem, if we get $I=\mu_{*}$ then $\mu$ is a weakly prime fuzzy ideal. Conversely, let $\mu$ is a weakly prime fuzzy ideal. Firstly we show that $|\operatorname{Im} \mu|=2$. Assume that $|\operatorname{Im} \mu| \geq 3$. Let $\mu(1)=k$. Since $\mu(0)=1$ then there exists $0 \neq r \in R$ such that $\mu(r)=m$ and $k<m<1$.

For fuzzy points $1_{m}, r_{1}$ of $R, 1_{m} \cdot r_{1}=r_{m \wedge 1}=r_{m} \in \mu$ because $\mu(r)=m \geq m=r_{m}(r)$. It is easy to see that $1_{m} \cdot r_{1} \neq 0_{t}$ for all $t \in(0,1]$. Since $\mu$ is a weakly prime fuzzy ideal and $0_{t} \neq 1_{m} \cdot r_{1}=r_{m} \in \mu$ then $1_{m} \in \mu$ or $r_{1} \in \mu$. However this contradicts that $1_{m}(1)=m>k=\mu(1)$ and $r_{1}(r)=1>m=\mu(r)$. Hence $|\operatorname{Im} \mu|=2$.

Now, we prove that $\mu_{*}$ is a weakly prime ideal. Assume that $0 \neq x y \in \mu_{*}$ and $x, y \notin \mu_{*}$. Since $\mu(0)=1$ then for all $t \in(0,1], t \leq \mu(x y)=\mu(0)=1$, so $0_{t} \neq(x y)_{t} \in \mu$. Since $\mu$ is a weakly prime fuzzy ideal then $x_{t} \in \mu$ or $y_{t} \in \mu$. For all $t \in(0,1], x_{t}(x)=t \leq \mu(x)$ or $y_{t}(y)=t \leq \mu(y)$. Especially, for $t=1, \mu(x)=1$ or $\mu(y)=1$ so $x \in \mu_{*}$ or $y \in \mu_{*}$ which is a contradiction by our assumption. Hence $\mu_{*}$ is a weakly prime ideal.

Lemma 2.8 [6] Let $A, B$ be fuzzy ideals of $R$ then for $t \in L A_{t} B_{t}=(A B)_{t}$.

Theorem 2.9 Let $\mu$ be a fuzzy ideals of $R$. Then $\mu$ is a weakly prime fuzzy ideal if and only if $0_{t} \neq A B \subseteq \mu$ implies $A \subseteq \mu$ or $B \subseteq \mu$ for any $A, B$ fuzzy ideals and for all $t \in(0, \mu(0)]$.

Proof. $\Leftarrow:$ Assume that for all $t \in(0, \mu(0)], 0_{t} \neq x_{r} y_{s} \in \mu$ where $x_{r}, y_{s}$ are any fuzzy points. Let nonconstant fuzzy subsets $A, B$ be defined by

$$
A(a)=\left\{\begin{array}{cc}
r & a \in<x> \\
0 & \text { otherwise }
\end{array} \text { and } B(b)=\left\{\begin{array}{cc}
s & b \in<y> \\
0 & \text { otherwise }
\end{array}\right. \text {. }\right.
$$

It is easy to see that $A, B$ are fuzzy ideals of $R$.

Case 1 If $z \in<x><y>$, then $A B(z)=\vee\{A(a) \wedge B(b): z=a b\}=r \wedge s \leq \mu(x y) \leq \mu(z)$.

Case 2 If $z \notin<x>\langle y>$, then $A B(z)=\vee\{A(a) \wedge B(b): z=a b\}=0 \leq \mu(z)$. Then we conclude that in all cases $A B \subseteq \mu$. By our assumption $0_{t} \neq x_{r} y_{s}$ then $x y \neq 0$. Thus $A B(x y)=r \wedge s \neq 0=0_{t}(x y)$ and we get that $0_{t} \neq A B$. By the hypothesis that $A \subseteq \mu$ or $B \subseteq \mu$ so $x_{r}(x)=r=A(x) \leq \mu(x)$ and $x_{r} \in \mu$ or $y_{s}(y)=s=B(y) \leq \mu(y)$ and $y_{s} \in \mu$. Hence we conclude that $\mu$ is a weakly prime fuzzy ideal.

$\Rightarrow$ : Now we show that $0_{t} \neq A B \subseteq \mu$ implies $A \subseteq \mu$ or $B \subseteq \mu$ for any $A, B$ fuzzy ideals and for all $t \in(0, \mu(0)]$. Let $0_{t} \neq A B \subseteq \mu$. Then there exists $r \in R$ such that $0_{t}(r) \neq A B(r)$.

Case 1 If $r=0$ for all $t \in(0, \mu(0)]$, then $0_{t}(0)=t \neq A B(0)$ and it must be $A B(0)=0$. Thus $A B=0_{R}$ and $A=0_{R}$ or $B=0_{R}$. Hence we have $A \subseteq \mu$ or $B \subseteq \mu$. 
Case 2 If $r \neq 0$ for some $t_{i} \in(0, \mu(0)]$, then $0_{t_{i}}(r)=0 \neq A B(r)$. If there exists any $0 \neq r \in R$ such that $0_{t_{i}}(r) \neq A B(r)$ then we conclude that for all $t \in(0, \mu(0)], 0_{t}(r)=0 \neq A B(r)$. Let $A B(r)=s \neq 0$ then $(A B)_{s} \neq\{0\}$. By Theorem 2.4, since $\mu$ is weakly prime fuzzy ideal then $\mu_{s}$ is also weakly prime ideal. Thus $0 \neq(A B)_{s}=A_{s} B_{s} \subseteq \mu_{s}$ and $A_{s} \subseteq \mu_{s}$ or $0 \neq(A B)_{s}=A_{s} B_{s} \subseteq \mu_{s}$. Hence $A \subseteq \mu$ or $B \subseteq \mu$.

Definition 2.10 (1) Let $\mu$ and $\alpha$ be two fuzzy ideals of $R$. The cartesian product of $\mu$ and $\alpha$ is defined by $\mu \times \alpha$ such that $(\mu \times \alpha)(x, y)=\mu(x) \wedge \alpha(y)$ [4].

(2) Let $\mu$ and $\alpha$ be two fuzzy ideals of $R$. If $\left(x_{r}, y_{s}\right) \in \mu \times \alpha$ for any fuzzy points $\mathrm{x}_{r}, \mathrm{y}_{s}$ of $R$ then $x_{r} \in \mu$ and $y_{s} \in \alpha$ so $r \wedge s \leq \mu \times \alpha(x, y)=\mu(x) \wedge \alpha(y)$.

Note that if $\mu$ and $\alpha$ are fuzzy ideals of $R$ then $\mu \times \alpha$ is a fuzzy ideal of $R \times R$.

Theorem 2.11 Let $R_{1}$ and $R_{2}$ be rings and $\mu_{1}$ and $\mu_{2}$ be weakly prime fuzzy ideals, respectively. Then $\mu_{1} \times \lambda_{R_{2}}$ and $\lambda_{R_{1}} \times \mu_{2}$ are weakly prime fuzzy ideals of $R_{1} \times R_{2}$.

Proof. It is easy to see that $\mu_{1} \times \lambda_{R_{2}}$ and $\lambda_{R_{1}} \times \mu_{2}$ are fuzzy ideals of $R_{1} \times R_{2}$. Let $\left(x_{r}, y_{s}\right),\left(z_{k}, t_{v}\right)$ be fuzzy points of $R_{1} \times R_{2}$ such that $\left(x_{r}, y_{s}\right)\left(z_{k}, t_{v}\right) \neq\left(0_{R_{1}}, 0_{R_{2}}\right)$. Then $x_{r} z_{k} \neq 0_{R_{1}}$ and $y_{s} t_{v} \neq 0_{R_{2}}$. $\left(x_{r}, y_{s}\right)\left(z_{k}, t_{v}\right) \in \mu_{1} \times \lambda_{R_{2}}$ then $\left(x_{r} z_{k}, y_{s} t_{v}\right) \in \mu_{1} \times \lambda_{R_{2}}$ so $x_{r} z_{k} \in \mu_{1}$ and $y_{s} t_{v} \in \lambda_{R_{2}}$. Since $\mu_{1}$ is weakly prime fuzzy ideal and $x_{r} z_{k} \neq 0_{R_{1}}$ then $x_{r} \in \mu_{1}$ or $z_{k} \in \mu_{1}$. Thus $\left(x_{r}, y_{s}\right) \in \mu_{1} \times \lambda_{R_{2}}$ or $\left(z_{k}, t_{v}\right) \in \mu_{1} \times \lambda_{R_{2}}$.

Similary, it can be easily shown that $\lambda_{R_{1}} \times \mu_{2}$ is a weakly prime fuzzy ideal of $R_{1} \times R_{2}$.

Definition 2.12 Let $\mu$ be a nonconstant fuzzy ideal of $R$. If $\mu(x y)=\mu(x)$ or $\mu(x y)=\mu(y)$ for $x y \neq 0$ then $\mu$ is called partial weakly prime fuzzy ideal.

Theorem 2.13 If $\mu$ is a weakly prime fuzzy ideal then $\mu$ is a partial weakly prime fuzzy ideal.

Proof. Assume that $\mu$ is a weakly prime fuzzy ideal and $x y \neq 0$.

If $\mu(x y)=0$ then $\mu(x) \leq \mu(x y)=0$ so $\mu(x)=\mu(x y)=0$.

If $\mu(x y) \neq 0$ then there exists $s \in(0, \mu(0)]$ such that $s=\mu(x y)$. Since $x y \neq 0$ then $(x y)_{s} \neq 0_{t}$ for all $t \in(0, \mu(0)]$. Because $0_{t} \neq(x y)_{s} \in \mu$ and $\mu$ is a weakly prime fuzzy ideal then $x_{s} \in \mu$ or $y_{s} \in \mu$. Thus $\mu(x y)=s \leq \mu(x)$ or $\mu(x y)=s \leq \mu(y)$ so $\mu(x y)=\mu(x)$ or $\mu(x y)=\mu(y)$.

The following example shows the converse of the theorem is not true.

Example 2.14 Let $R=Z \times Z$. Define the fuzzy ideal $\mu$ of $R$ by 


$$
\mu(a)=\left\{\begin{array}{cc}
1, & \text { if } a \in 0 \times Z \\
1 / 2, & \text { if } a \in 2 \times Z \backslash 0 \times Z . \\
0, & \text { if } a \in Z \times Z \backslash 2 \times Z
\end{array}\right.
$$

It is easy to prove that $\mu$ is a partial weakly prime fuzzy ideal . However $\mu$ is not weakly prime fuzzy ideal. Assume that $a=(2, r)$ and $b=(3, s)$. For fuzzy points of $R a_{\frac{2}{3}}=(2, r)_{\frac{2}{3}}$ and $b_{\frac{1}{3}}=(3, s)_{\frac{1}{3}}$, we get that $a_{\frac{2}{3}} \notin \mu$ and $b_{\frac{1}{3}} \notin \mu$. But $0_{t} \neq a_{\frac{2}{3}} \cdot b_{\frac{1}{3}}=(a b)_{\frac{1}{3}}=(6, r s)_{\frac{1}{3}} \in \mu$. Hence $\mu$ is not weakly prime fuzzy ideal.

Theorem 2.15 Let $\mu$ be a nonconstant fuzzy ideal of $R$. Then $\mu$ is a partial weakly prime fuzzy ideal if and only if $\mu_{t}$ is a weakly prime ideal of $R$ for all $t \in(0, \mu(0)]$.

Proof. Assume that $\mu$ is a partial weakly prime fuzzy ideal. Let $0 \neq x y \in \mu_{t}$. Then $\mu(x y) \geq t$. Because $\mu$ is a partial weakly prime then $\mu(x)=\mu(x y) \geq t$ and $x \in \mu_{t}$ or $\mu(y)=\mu(x y) \geq t$ and $y \in \mu_{t}$. Let $\mu_{t}$ be a weakly prime ideal. For $x y \neq 0$, let $\mu(x y)=s$.

Case 1 If $s=0$, then $\mu(x) \leq \mu(x y)=0$ and $\mu(x y)=\mu(x)=0$ or $\mu(y) \leq \mu(x y)=0$ and $\mu(x y)=\mu(y)=0$.

Case2 If $s \neq 0$, then $0 \neq x y \in \mu_{s}$. Since $\mu_{s}$ is weakly prime ideal then $x \in \mu_{s}$ or $y \in \mu_{s}$ thus $\mu(x) \geq s=\mu(x y)$ and $\mu(x y)=\mu(x)$ or $\mu(y) \geq s=\mu(x y)$ and $\mu(x y)=\mu(y)$.

Theorem 2.16 Let $f$ be an injective ring homomorphism from $R$ to $S$. If $\xi$ is a partial weakly prime fuzzy ideal of $S$ then $f^{-1}(\xi)$ is a partial weakly prime fuzzy ideal of $R$.

Proof. Let $0 \neq r u$ where $r, u \in R . f^{-1}(\xi)(r u)=\xi(f(r u))=\xi(f(r) f(u))$. Since $f$ is $1-1$ then $f(r u) \neq 0$. Because $\xi$ is partial weakly prime then $\xi(f(r u))=\xi(f(r) f(u))=\xi(f(r))$ so $f^{-1}(\xi)(r u)=f^{-1}(\xi)(r)$ or $\xi(f(r u))=\xi(f(r) f(u))=\xi(f(u))$ so $f^{-1}(\xi)(r u)=f^{-1}(\xi)(u)$. Hence we conclude that $f^{-1}(\xi)$ is a partial weakly prime fuzzy ideal.

Theorem 2.17 Let $f: R \rightarrow S$ be a surjective ring homorphism. If $\mu$ is a partial weakly prime fuzzy ideal of $R$ which is constant on $\operatorname{Kerf}$ then $f(\mu)$ is a partial weakly prime fuzzy ideal of $S$.

Proof. Assume that $x y \neq 0$ where $x, y \in S$. Since $f$ is an epimorphism then there exist $r, u \in R$ such that $f(r)=x, f(u)=s$. Because $f$ is constant on Kerf so

$$
f(\mu)(x y)=f(\mu)(f(r) f(u))=f(\mu)(f(r u))=\mu(r u) .
$$

If $r u=0$ then $f(0)=0=f(r u)=f(r) f(u)=x y$, which is a contradiction. Then $r u \neq 0$. Since $\mu$ is a partial weakly prime fuzzy ideal then

$$
\begin{gathered}
f(\mu)(x y)=\mu(r u)=\mu(r)=f(\mu) f(r)=f(\mu)(x) \text { or } \\
f(\mu)(x y)=\mu(r u)=\mu(u)=f(\mu) f(u)=f(\mu)(y) .
\end{gathered}
$$


Hence we get that $f(\mu)$ is a partial weakly prime fuzzy ideal.

\section{Conclusions}

In this paper, we have characterized weakly prime fuzzy ideals. Also the notions of partial weakly prime fuzzy ideals and their properties are proposed. Furthermore, we have given generalization of definitions of weakly prime fuzzy ideals.

To extend this study, one could study other algebraic structures and do some further studies of their properties.

\section{References}

[1] Anderson D. D. and Smith E., "Weakly prime ideals”, Houston J. Math. 29(4) (2003) : 831-840.

[2] Atani S. E. and Farzalipour F., “On weakly primary ideals”, Georgian Math. J. 12(3) (2005) : 423-429.

[3] Dixit V.N., Kumar R. and Ajmal N., "Fuzzy ideals and fuzzy prime ideals of a ring”, Fuzzy Sets and Systems 44 (1991) : 127-138.

[4] Ersoy B.A., "A Generalization of Cartesian Product of Fuzzy Subgroups and Ideals”, Journal of Applied Sciences 3 (2003) : 100-102.

[5] Liu W.J., "Fuzzy invariant subgroups and fuzzy ideals”, Fuzzy Sets and Systems 8 (1982) : 133-139.

[6] Martinez L., "Fuzzy subgroups of fuzzy groups and fuzzy ideals of fuzzy rings”, J. Fuzzy Math. 3 (1995) : 833-849.

[7] Mukherjee T.K. and Sen M.K., "Prime fuzzy ideals in rings”, Fuzzy Sets and Systems 32 (1989) : 337-341.

[8] Zadeh L.A., "Fuzzy sets”, Inform and Control 8 (1965) : 338-353. 